\title{
A novel and efficient method to prepare 2-aryl- tetrahydrofuran-2-ylphosphonic acids
}

\author{
Vsevolod V. Komissarov ${ }^{1}$, Anatoly M. Kritzyn ${ }^{1}$ and Jouko J. Vepsäläinen ${ }^{* 2}$
}

\section{Full Research Paper}

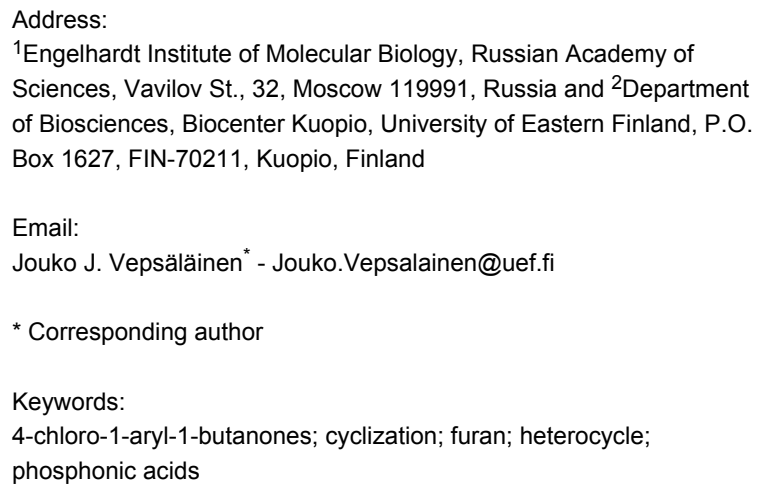

Beilstein J. Org. Chem. 2010, 6, No. 63. doi:10.3762/bjoc.6.63

Received: 21 March 2010

Accepted: 18 May 2010

Published: 09 June 2010

Associate Editor: J. A. Porco Jr

(c) 2010 Komissarov et al; licensee Beilstein-Institut. License and terms: see end of document.

\begin{abstract}
A novel one-pot method was developed for the synthesis of the title compounds starting from 4-chloro-1-aryl-1-butanones 1, phosphorus trichloride and acetic acid. The end products 2 were obtained in 20-94\% yield. The cyclization step under acidic conditions probably occurs as a result of anchimeric assistance of the phosphonic acid group.
\end{abstract}

\section{Introduction}

Our laboratory has conducted systematic evaluation of polymethylene derivatives of nucleic bases with various terminal functional groups [1-4]. As a part of our ongoing project, it seemed important to synthesize compounds with phosphonate and phenyl groups at one end of the hydrocarbon chain and a nucleic base residue at the other end. Previously, it was shown that compounds with similar structures inhibit purine nucleoside phosphorylase and thymidine phosphorylase, enzymes which have been targeted in a number of serious diseases [5-7].

Recently, we prepared nucleic base derivatives with a terminal Ph-CO-group by alkylation methods starting from 4-chloro-1aryl-1-butanones 1 [8]. According to the studies of Conant et al. $[9,10]$ conducted about 90 years ago, the target phenylvinyl- phosphonic acids 3 could be obtained from $\mathbf{1}$ after treatment with $\mathrm{PCl}_{3}$ and $\mathrm{AcOH}$, followed by the elimination of water (Figure 1). After the reduction of the double bond [11], these compounds could then be used for nucleic bases alkylation as described earlier.

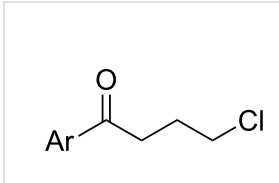

1

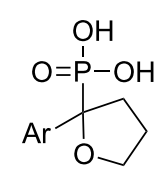

2<smiles>O=P(O)(O)C([Al])=CCCCl</smiles>

3
Figure 1: Treatment of 4-chloro-1-aryl-1-butanones 1 with $\mathrm{PCl}_{3} / \mathrm{AcOH}$ lead to unexpected cyclic product 2 instead of the expected alkene 3. 


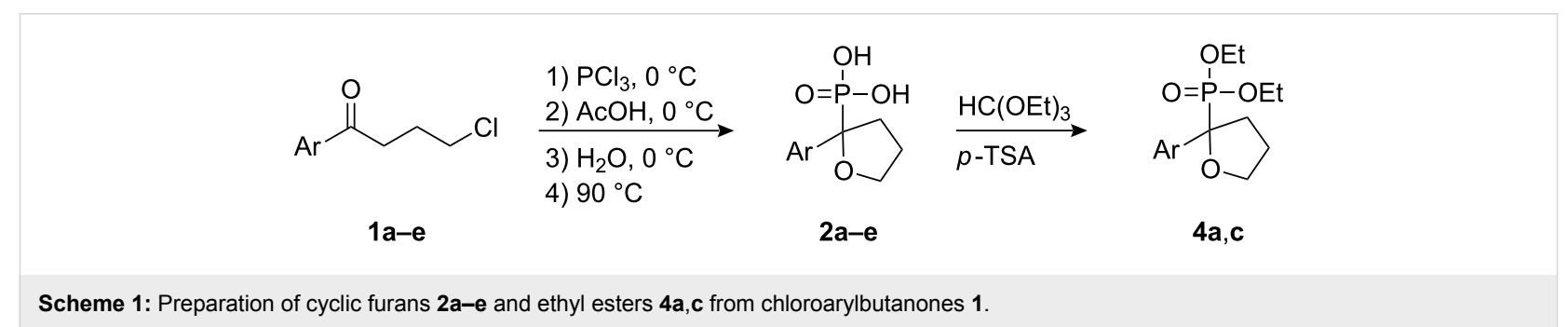

\section{Results and Discussion}

In this study, we employed the above noted reaction sequence with our model compound, 4-chloro-1-phenyl-1-butanone (1a) [5]. However, the only product formed in good yield was 2-phenyltetrahydrofuran-2-ylphosphonic acid (2a) instead of the expected acyclic product. Previously, to obtain phenylvinylphosphonic acids 3, Conant [9] heated the reaction mixture under atmospheric pressure at high temperature $\left(\sim 280{ }^{\circ} \mathrm{C}\right)$. Initially, we also followed this procedure, but soon realized that the yield of phosphonic acid $\mathbf{2 a}$ was significantly higher under the milder conditions shown in Scheme 1. The yields of the prepared cyclic compounds $\mathbf{2} \mathbf{b}-\mathbf{e}$ are summarized in Table 1.

\begin{tabular}{|c|c|c|}
\hline Aryl group & Product & Yield [\%] \\
\hline & $2 a$ & 94 \\
\hline & $2 b$ & 59 \\
\hline & $2 c$ & 83 \\
\hline & $2 d$ & 57 \\
\hline & $2 e$ & $20^{a}$ \\
\hline
\end{tabular}

alsolated via the ammonium salt.

The synthesized phosphonic acids 2 can be easily converted into diethyl esters 4 by refluxing in triethyl orthoformate in the presence of $p$-TSA [12]. Previously, these esters 4 were prepared directly from $\mathbf{1}$ - $-\mathbf{d}$ by refluxing in an excess of $\mathrm{P}(\mathrm{OEt})_{3}$. However, this procedure provides high yields of 2-aryltetrahydrofuran-2-ylphosphonic acid esters $\mathbf{4}$ only if the starting ketones have electron donating substituents at the paraposition of the aromatic ring $[13,14]$.

The structures of phosphonic acids 2 were established from MS and NMR spectral data. The cyclic structures of the end products were verified not only from molecular ion peaks in the mass spectra but also from ${ }^{1} \mathrm{H}$ NMR data, in which three $\mathrm{CH}_{2}$ groups give rise to six chemical shifts. This phenomenon exists if a chiral center (sometimes a prochiral center) is near a $\mathrm{CH}_{2}-$ group or when a $\mathrm{CH}_{2}$ group is a part of a cyclic structure. In this case, the chemical shift difference between the furan ring $\mathrm{CH}_{2}$ protons were $0.1-0.5 \mathrm{ppm}$, clear evidence for the existence of a cyclic structure. The complicated but characteristic ${ }^{1} \mathrm{H}$ NMR spectra were analyzed by PERCH software [15]. In addition, ${ }^{13} \mathrm{C}$ NMR spectra confirmed the presence of the tetrahydrofuran ring system since characteristic ${ }^{1-3} J_{\mathrm{CP}}$ coupling constants were found for all four $\mathrm{CH}_{2}$ carbons at $\sim 84, \sim 69, \sim 36$, and $\sim 26$ ppm. ${ }^{31} \mathrm{P}$ NMR signals of the phosphonic acids 2 were at ca 23 ppm.

In order to understand the mechanism of the reaction, we conducted the reactions of $\mathbf{1 a}$ and $\mathrm{PCl}_{3}$ in the absence of $\mathrm{AcOH}$ and with phosphorous acid in $\mathrm{AcOH}$ solution. In the first case, there was no evidence of that any reaction had occurred, whereas in the second case cyclization was observed only under reflux conditions; however, the yield of $\mathbf{2 a}$ was extremely low. Accordingly, in the initial step either $\mathrm{PCl}_{3}$ reacts with ketone 1 to form the adduct $\mathbf{5}$, as proposed by Conant [9], which is then converted to intermediate $\mathbf{6}$ via $\mathbf{5 a}$ by the addition of $\mathrm{AcOH}$, or alternatively, $\mathrm{HOPCl}_{2}$ (and/or $\left.(\mathrm{HO})_{2} \mathrm{PCl}\right)$, formed after acetic acid addition to the mixture, reacts directly with the ketone 1 to produce intermediate 6 as shown in Scheme 2.

The intermediate 6 rapidly forms ring 8 possibly via intermediate 7 . However, the cyclization reaction in which hydroxyl group and chlorine atom are sterically near to each other are normally conducted under basic conditions [16] or at elevated temperatures [17]. A plausible explanation for the cyclization occurring at room temperature without an alkoxide intermediate would be to invoke anchimeric assistance of the $\mathrm{P}=\mathrm{O}$ group, which could serve as a general base to facilitate cyclization leading to the formation of 7 [18].

The length of hydrocarbon chain is also of major importance, since cyclic tetrahydropyran derivatives are not formed in the case of arylpentanones $\mathbf{9 a , b}$ under the same conditions as those used for the synthesis of furans 2 (Scheme 3). According to the 


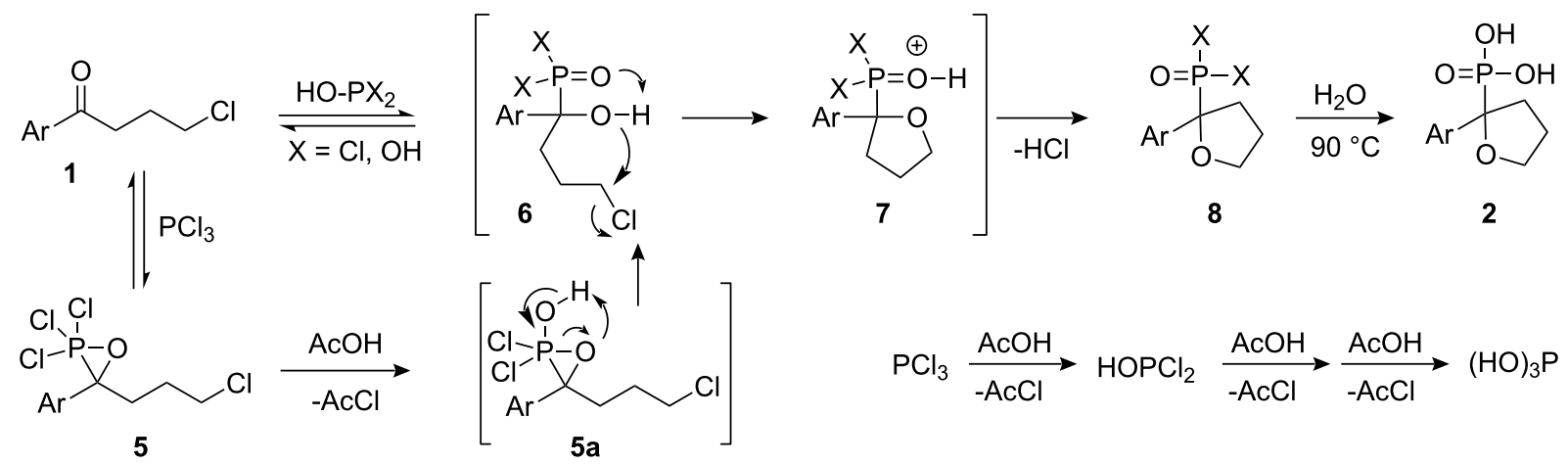

Scheme 2: Proposed reaction mechanism for the cyclization reaction.

NMR spectra, the only phosphorus containing products in the reaction mixtures were the corresponding $\alpha$-hydroxyphosphonic acids 10. Similarly, acid 10c is formed from phenylbutanone $4 \mathbf{c}$ in $41 \%$ yield.<smiles>[R]CCCC(=O)c1ccc([X])cc1</smiles>

1) $\mathrm{PCl}_{3}, 0^{\circ} \mathrm{C}$

2) $\mathrm{AcOH}, 0^{\circ} \mathrm{C}$

3) $\mathrm{H}_{2} \mathrm{O}, 0^{\circ} \mathrm{C}$ 4) $90^{\circ} \mathrm{C}$

9a $\mathrm{R}=\mathrm{CH}_{2} \mathrm{Cl}, \mathrm{X}=\mathrm{H}$

9b R $=\mathrm{CH}_{2} \mathrm{Cl}, \mathrm{X}=\mathrm{F}$

9c $\mathrm{R}=\mathrm{X}=\mathrm{H}$

Scheme 3: Acyclic products are obtained from pentanones $\mathbf{9 a , b}$ and butanone $9 \mathrm{c}$

In summary, a direct and efficient route was developed for the synthesis of 2-aryltetrahydrofuran-2-ylphosphonic acids - compounds containing a relatively exotic motif - via a one-pot experimental procedure from 4-chloro-1-aryl-1-butanones. The cyclic structure of the synthesized compounds was reliably established by means of various NMR experiments. A mechanism for the cyclization is proposed and it is suggested, that the crucial feature of the reaction is the anchimeric assistance of the $\mathrm{P}=\mathrm{O}$ group. We have also demonstrated that the tetrahydropyran analogs of the title phosphonic acids were not formed under similar conditions.

\section{Experimental}

General remarks. ${ }^{1} \mathrm{H},{ }^{13} \mathrm{C}$ and ${ }^{31} \mathrm{P}$ NMR spectra were recorded on a Bruker Avance 500 DRX instrument at $500 \mathrm{MHz}, 125$ $\mathrm{MHz}$ and $162 \mathrm{MHz}$, respectively. Chemical shifts $(\delta)$ are reported in ppm relative to tetramethylsilane as internal standard for protons and carbon atoms, and to $\mathrm{H}_{3} \mathrm{PO}_{4}(85 \%)$ for phosphorus chemical shifts. Exact chemical shifts and coupling constants for protons were calculated using PERCH software
[15]. Mass spectra were obtained on an Applied Biosystems/ MDS Sciex QSTAR XL spectrometer using ESI technique.

General procedure for the synthesis of compounds 2a-e: $\mathrm{PCl}_{3}(1.2 \mathrm{~mL}, 13.75 \mathrm{mmol})$ was added dropwise to 4-chloro-1aryl-1-butanone $(10 \mathrm{mmol})$ with stirring at $0{ }^{\circ} \mathrm{C}$. Cooling was removed and the reaction mixture stirred at room temperature for $30 \mathrm{~min}$ followed by dropwise addition of glacial acetic acid $(1.72 \mathrm{~mL}, 30 \mathrm{mmol})$ with stirring at $0{ }^{\circ} \mathrm{C}$. Stirring was then continued without cooling for $20 \mathrm{~h}$, ice $(50 \mathrm{~g})$ was added and the reaction mixture heated slowly over a water bath to $90{ }^{\circ} \mathrm{C}$. After $40 \mathrm{~min}$ at this temperature, the solvents were evaporated in vacuo and the resulting oil was re-evaporated with water $(3 \times$ $20 \mathrm{~mL})$. The crystalline residue was washed with cold water $(20$ $\mathrm{mL})$ and benzene $(40 \mathrm{~mL})$. The residue was dried under vacuum $\left(\mathrm{P}_{2} \mathrm{O}_{5}\right.$ and paraffin) to yield the target acids $\mathbf{2 a - d}$ in $57-94 \%$ yield.

2-Phenyltetrahydrofuran-2-ylphosphonic acid (2a): ${ }^{1} \mathrm{H} \mathrm{NMR}\left(\mathrm{DMSO}-d_{6}\right): \delta_{\mathrm{H}} 7.5(2 \mathrm{H}, \mathrm{bs}), 7.450\left(2 \mathrm{H}, \mathrm{d},{ }^{3} J_{\mathrm{HH}}=\right.$ $7.80 \mathrm{~Hz}), 7.288\left(2 \mathrm{H}, \mathrm{dd},{ }^{3} J_{\mathrm{HH}}=7.80,7.39 \mathrm{~Hz}\right), 7.203(1 \mathrm{H}, \mathrm{dd})$, $3.923\left(1 \mathrm{H}, \mathrm{ddd},{ }^{2} J_{\mathrm{HH}}=-7.84 \mathrm{~Hz},{ }^{3} J_{\mathrm{HH}}=7.11,6.90\right), 3.847$ $\left(1 \mathrm{H}, \mathrm{ddd},{ }^{2} J_{\mathrm{HH}}=-7.84 \mathrm{~Hz},{ }^{3} J_{\mathrm{HH}}=7.37,5.42 \mathrm{~Hz}\right), 2.599(1 \mathrm{H}$, dddd, ${ }^{3} J_{\mathrm{HP}}=15.58 \mathrm{~Hz},{ }^{2} J_{\mathrm{HH}}=-12.54 \mathrm{~Hz},{ }^{3} J_{\mathrm{HH}}=8.13,7.82$ $\mathrm{Hz}), 2.137\left(1 \mathrm{H}, \mathrm{dddd},{ }^{3} J_{\mathrm{HP}}=10.97 \mathrm{~Hz},{ }^{2} J_{\mathrm{HH}}=-12.54 \mathrm{~Hz}\right.$, $\left.{ }^{3} J_{\mathrm{HH}}=7.77,5.12 \mathrm{~Hz}\right), 1.980\left(\mathrm{ddddd},{ }^{2} J_{\mathrm{HH}}=-11.74 \mathrm{~Hz},{ }^{3} J_{\mathrm{HH}}=\right.$ $8.13,6.90,5.42,5.12$ ), 1.660 (ddddd, ${ }^{3} J_{\mathrm{HH}}=7.82,7.77,7.37$, 7.11); ${ }^{13} \mathrm{C}$ NMR (DMSO- $\left.d_{6}\right): \delta_{\mathrm{C}} 142.4 \mathrm{~s}, 127.3 \mathrm{~s}, 126.4 \mathrm{~s}$, $126.3 \mathrm{~s}, 83.6\left(\mathrm{~d}, J_{\mathrm{CP}}=167.0 \mathrm{~Hz}\right), 68.4\left(\mathrm{~d}, J_{\mathrm{CP}}=6.4 \mathrm{~Hz}\right), 35.7 \mathrm{~s}$, $25.5\left(\mathrm{~d}, J_{\mathrm{CP}}=4.8 \mathrm{~Hz}\right) ;{ }^{31} \mathrm{P} \mathrm{NMR}\left(\mathrm{DMSO}-d_{6}\right): \delta_{\mathrm{P}} 23.11$; MS (ESI): $m / z 227\left[\mathrm{M}^{+}-\mathrm{H}\right]$.

\section{2-(4-Methylphenyl)tetrahydrofuran-2-ylphosphonic acid} (2b): ${ }^{1} \mathrm{H}$ NMR (DMSO- $\left.d_{6}\right): \delta_{\mathrm{H}} 8.6(2 \mathrm{H}, \mathrm{bs}), 7.326\left(2 \mathrm{H}, \mathrm{d},{ }^{3} J_{\mathrm{HH}}\right.$ $=7.87 \mathrm{~Hz}), 7.089(2 \mathrm{H}, \mathrm{d}), 3.906\left(1 \mathrm{H}, \mathrm{ddd},{ }^{2} J_{\mathrm{HH}}=-7.81 \mathrm{~Hz}\right.$, $\left.{ }^{3} J_{\mathrm{HH}}=7.13,6.94\right), 3.831\left(1 \mathrm{H}, \mathrm{ddd},{ }^{2} J_{\mathrm{HH}}=-7.81 \mathrm{~Hz},{ }^{3} J_{\mathrm{HH}}=\right.$ 
7.40, $5.36 \mathrm{~Hz}), 2.270(3 \mathrm{H}, \mathrm{s}), 2.567\left(1 \mathrm{H}, \mathrm{dddd},{ }^{3} J_{\mathrm{HP}}=15.51\right.$ $\left.\mathrm{Hz},{ }^{2} J_{\mathrm{HH}}=-12.51 \mathrm{~Hz},{ }^{3} J_{\mathrm{HH}}=8.09,7.96 \mathrm{~Hz}\right), 2.110(1 \mathrm{H}$, dddd, $\left.{ }^{3} J_{\mathrm{HP}}=10.78 \mathrm{~Hz},{ }^{2} J_{\mathrm{HH}}=-12.51 \mathrm{~Hz},{ }^{3} J_{\mathrm{HH}}=7.71,4.98 \mathrm{~Hz}\right)$, $1.964\left(\right.$ ddddd, $\left.{ }^{2} J_{\mathrm{HH}}=-11.73 \mathrm{~Hz},{ }^{3} J_{\mathrm{HH}}=8.09,6.94,5.36,4.98\right)$, $1.648\left(\mathrm{ddddd},{ }^{3} J_{\mathrm{HH}}=7.96,7.70,7.40,7.13\right) ;{ }^{13} \mathrm{C} \mathrm{NMR}$ $\left(\mathrm{DMSO}-d_{6}\right): \delta_{\mathrm{C}} 139.7\left(\mathrm{~d}, J_{\mathrm{CP}}=5.6 \mathrm{~Hz}\right), 135.9\left(\mathrm{~d}, J_{\mathrm{CP}}=2.4\right.$ $\mathrm{Hz}), 128.4 \mathrm{~s}, 126.6\left(\mathrm{~d}, J_{\mathrm{CP}}=3.2 \mathrm{~Hz}\right), 83.9\left(\mathrm{~d}, J_{\mathrm{CP}}=169.0 \mathrm{~Hz}\right)$, $68.8\left(\mathrm{~d}, J_{\mathrm{CP}}=6.4 \mathrm{~Hz}\right), 36.0\left(\mathrm{~d}, J_{\mathrm{CP}}=2.4 \mathrm{~Hz}\right), 25.9\left(\mathrm{~d}, J_{\mathrm{CP}}=4.8\right.$ $\mathrm{Hz}), 20.9 \mathrm{~s} ;{ }^{31} \mathrm{P}$ NMR (DMSO- $\left.d_{6}\right): \delta_{\mathrm{P}} 23.56$; MS (ESI): $\mathrm{m} / \mathrm{z}$ $241\left[\mathrm{M}^{+}-\mathrm{H}\right]$.

2-(4-Fluorophenyl)tetrahydrofuran-2-ylphosphonic acid (2c): ${ }^{1} \mathrm{H}$ NMR (DMSO- $\left.d_{6}\right): \delta_{\mathrm{H}} 8.3(2 \mathrm{H}, \mathrm{bs}), 7.463(2 \mathrm{H}, \mathrm{dd}$, $\left.{ }^{3} J_{\mathrm{HH}}=8.71 \mathrm{~Hz},{ }^{4} J_{\mathrm{HF}}=5.58 \mathrm{~Hz}\right), 7.111\left(2 \mathrm{H}, \mathrm{dd},{ }^{3} J_{\mathrm{HF}}=8.01\right.$ $\mathrm{Hz}), 3.919\left(1 \mathrm{H}, \mathrm{ddd},{ }^{2} J_{\mathrm{HH}}=-7.87 \mathrm{~Hz},{ }^{3} J_{\mathrm{HH}}=7.16,6.90 \mathrm{~Hz}\right)$, $3.854\left(1 \mathrm{H}, \mathrm{ddd},{ }^{2} J_{\mathrm{HH}}=-7.87 \mathrm{~Hz},{ }^{3} J_{\mathrm{HH}}=7.39,5.36 \mathrm{~Hz}\right), 2.588$ $\left(1 \mathrm{H}, \mathrm{dddd},{ }^{3} J_{\mathrm{HP}}=15.41 \mathrm{~Hz},{ }^{2} J_{\mathrm{HH}}=-12.58 \mathrm{~Hz},{ }^{3} J_{\mathrm{HH}}=8.11\right.$, $7.90 \mathrm{~Hz}), 2.126\left(1 \mathrm{H}\right.$, dddd, ${ }^{3} J_{\mathrm{HP}}=10.86 \mathrm{~Hz},{ }^{2} J_{\mathrm{HH}}=-12.58 \mathrm{~Hz}$, $\left.{ }^{3} J_{\mathrm{HH}}=7.75,5.07 \mathrm{~Hz}\right), 1.986\left(\mathrm{ddddd},{ }^{2} J_{\mathrm{HH}}=-11.77 \mathrm{~Hz},{ }^{3} J_{\mathrm{HH}}=\right.$ $8.11,6.90,5.36,5.07), 1.667$ (ddddd, ${ }^{3} J_{\mathrm{HH}}=7.90,7.75,7.39$, 7.16); ${ }^{13} \mathrm{C}$ NMR (DMSO- $\left.d_{6}\right): \delta_{\mathrm{C}} 161.9\left(\mathrm{~d}, J_{\mathrm{CF}}=242.6 \mathrm{~Hz}\right)$, 138.9 s, 128.9 (d, $J=4.6 \mathrm{~Hz}), 114.6(\mathrm{~d}, J=21.4 \mathrm{~Hz}), 83.9$ (d, $\left.J_{\mathrm{CP}}=169.4 \mathrm{~Hz}\right), 69.2\left(\mathrm{~d}, J_{\mathrm{CP}}=6.1 \mathrm{~Hz}\right), 36.2 \mathrm{~s}, 26.1\left(\mathrm{~d}, J_{\mathrm{CP}}=\right.$ $4.6 \mathrm{~Hz}) ;{ }^{31} \mathrm{P}$ NMR (DMSO- $\left.d_{6}\right): \delta_{\mathrm{P}} 22.72$; MS (ESI): $m / z 245$ $\left[\mathrm{M}^{+}-\mathrm{H}\right]$.

2-(4-Methoxyphenyl)tetrahydrofuran-2-ylphosphonic acid (2d): ${ }^{1} \mathrm{H}$ NMR (DMSO- $\left.d_{6}\right): \delta_{\mathrm{H}} 8.3(2 \mathrm{H}, \mathrm{bs}), 7.357\left(2 \mathrm{H}, \mathrm{d},{ }^{3} J_{\mathrm{HH}}\right.$ $=8.55 \mathrm{~Hz}), 6.857(2 \mathrm{H}, \mathrm{d}), 3.909\left(1 \mathrm{H}, \mathrm{dt},{ }^{2} J_{\mathrm{HH}}=-7.77 \mathrm{~Hz}\right.$, $\left.{ }^{3} J_{\mathrm{HH}}=7.06\right), 3.837\left(1 \mathrm{H}, \mathrm{ddd},{ }^{2} J_{\mathrm{HH}}=-7.77 \mathrm{~Hz},{ }^{3} J_{\mathrm{HH}}=7.35\right.$, $5.33 \mathrm{~Hz}), 3.730(3 \mathrm{H}, \mathrm{s}), 2.562\left(1 \mathrm{H}, \mathrm{dddd},{ }^{3} J_{\mathrm{HP}}=15.32 \mathrm{~Hz}\right.$, $\left.{ }^{2} J_{\mathrm{HH}}=-12.48 \mathrm{~Hz},{ }^{3} J_{\mathrm{HH}}=8.06,8.04 \mathrm{~Hz}\right), 2.118(1 \mathrm{H}, \mathrm{dddd}$, $\left.{ }^{3} J_{\mathrm{HP}}=10.69 \mathrm{~Hz},{ }^{2} J_{\mathrm{HH}}=-12.48 \mathrm{~Hz},{ }^{3} J_{\mathrm{HH}}=7.64,4.98 \mathrm{~Hz}\right)$, $1.974\left(\mathrm{ddddd},{ }^{2} J_{\mathrm{HH}}=-11.70 \mathrm{~Hz},{ }^{3} J_{\mathrm{HH}}=8.04,7.06,5.33,4.98\right)$, $1.666\left(\mathrm{ddddd},{ }^{3} J_{\mathrm{HH}}=8.06,7.64,7.35,7.06\right) ;{ }^{13} \mathrm{C} \mathrm{NMR}$ (DMSO- $\left.d_{6}\right): \delta_{\mathrm{C}} 158.6 \mathrm{~s}, 134.5\left(\mathrm{~d}, J_{\mathrm{CP}}=4.8 \mathrm{~Hz}\right), 127.9 \mathrm{~s}, 113.3$ $\mathrm{s}, 83.7\left(\mathrm{~d}, J_{\mathrm{CP}}=169.5 \mathrm{~Hz}\right), 68.8\left(\mathrm{~d}, J_{\mathrm{CP}}=6.4 \mathrm{~Hz}\right), 55.4 \mathrm{~s}, 35.9$ s, $25.9\left(\mathrm{~d}, J_{\mathrm{CP}}=4.8 \mathrm{~Hz}\right) ;{ }^{31} \mathrm{P}$ NMR $\left(\mathrm{DMSO}-d_{6}\right): \delta_{\mathrm{P}} 23.74 ; \mathrm{MS}$ (ESI): $m / z 257\left[\mathrm{M}^{+}-\mathrm{H}\right]$.

2-(Naphthalen-2-yl)tetrahydrofuran-2-ylphosphonic acid (2e): After the solvents were evaporated, an excess of semisaturated methanolic ammonia was added and the mixture evaporated in vacuo. The crystalline product was washed with benzene $(70 \mathrm{~mL})$, the residue transferred to a separation funnel and $\mathrm{HCl}(15 \%, 50 \mathrm{~mL})$ and $\mathrm{CH}_{2} \mathrm{Cl}_{2}(50 \mathrm{~mL})$ added. The organic layer was separated and the aqueous phase extracted with $\mathrm{CH}_{2} \mathrm{Cl}_{2}(4 \times 50 \mathrm{~mL})$. The combined organic extracts were dried $\left(\mathrm{Na}_{2} \mathrm{SO}_{4}\right)$, evaporated in vacuo and the product recrystallized from benzene - heptane (2:1 mixture, $30 \mathrm{~mL})$ to give $\mathbf{2 e}$ : ${ }^{1} \mathrm{H}$ NMR (DMSO- $\left.d_{6}\right): \delta_{\mathrm{H}} 9.23(2 \mathrm{H}, \mathrm{bs}), 8.01(1 \mathrm{H}, \mathrm{s}), 7.90(1 \mathrm{H}$, d, $J=7.2 \mathrm{~Hz}), 7.85(2 \mathrm{H}, \mathrm{m}), 7.70(1 \mathrm{H}, \mathrm{d}, J=8.7 \mathrm{~Hz}), 7.46(2 \mathrm{H}$, $\mathrm{m}), 4.01(1 \mathrm{H}, \mathrm{m}), 3.92(1 \mathrm{H}, \mathrm{m}), 2.73(1 \mathrm{H}, \mathrm{m}), 2.26(1 \mathrm{H}, \mathrm{m})$, $2.00(1 \mathrm{H}, \mathrm{m}), 1.67(1 \mathrm{H}, \mathrm{m}) ;{ }^{13} \mathrm{C}$ NMR $\left(\mathrm{DMSO}-d_{6}\right): \delta_{\mathrm{C}} 140.4(\mathrm{~d}$, $\left.J_{\mathrm{CP}}=5.6 \mathrm{~Hz}\right), 132.7\left(\mathrm{~d}, J_{\mathrm{CP}}=2.4 \mathrm{~Hz}\right), 132.3\left(\mathrm{~d}, J_{\mathrm{CP}}=1.6 \mathrm{~Hz}\right)$, $128.1 \mathrm{~s}, 127.5 \mathrm{~s}, 127.0 \mathrm{~s}, 126.1 \mathrm{~s}, 125.8 \mathrm{~s}, 125.5\left(\mathrm{~d}, J_{\mathrm{CP}}=2.4\right.$ $\mathrm{Hz}), 124.9\left(\mathrm{~d}, J_{\mathrm{CP}}=5.6 \mathrm{~Hz}\right), 84.1\left(\mathrm{~d}, J_{\mathrm{CP}}=167.0 \mathrm{~Hz}\right), 68.8(\mathrm{~d}$, $\left.J_{\mathrm{CP}}=6.4 \mathrm{~Hz}\right), 35.8\left(\mathrm{~d}, J_{\mathrm{CP}}=3.2 \mathrm{~Hz}\right), 25.8\left(\mathrm{~d}, J_{\mathrm{CP}}=4.8 \mathrm{~Hz}\right)$; ${ }^{31} \mathrm{P}$ NMR (DMSO- $d_{6}$ ): $\delta_{\mathrm{P}} 22.98$; MS (ESI): $\mathrm{m} / z 277\left[\mathrm{M}^{+}-\mathrm{H}\right]$.

\section{Acknowledgements}

The authors would like to thank Ewen McDonald for language editing, Dr. Alex Khomutov, Engelhardt Institute of Molecular Biology, RAS, Russia, for critical remarks and helpful discussions, and PhoSciNet COST action (CM0802). This work was supported by Grants of the Russian Foundation for Basic Research (06-04-48716) and the Academy of Finland.

\section{References}

1. Makinsky, A. A.; Kritzyn, A. M.; Ul'janova, E. A.; Zakharova, O. D.; Nevinsky, G. A. Russ. J. Bioorg. Chem. 2000, 26, 662. doi:10.1007/BF02821834

2. Makinsky, A. A.; Kritzyn, A. M.; Ul'janova, E. A.; Zakharova, O. D.; Bugreev, D. V.; Nevinsky, G. A. Russ. J. Bioorg. Chem. 2001, 27, 167. doi:10.1023/A:1011377103793

3. Kritzyn, A. M.; Komissarov, V. V. Russ. J. Bioorg. Chem. 2004, 30, 436. doi:10.1023/B:RUBI.0000043786.12780.8a

4. Kritzyn, A. M.; Vepsalainen, J.; Komissarov, V. V. Russ. J. Bioorg. Chem. 2005, 31, 256. doi:10.1007/s11171-005-0035-3

5. Esteban-Gamboa, A.; Balzarini, J.; Esnouf, R.; De Clercq, E.; Camarasa, M.-J.; Perez-Perez, M.-J. J. Med. Chem. 2000, 43, 971. doi:10.1021/jm9911377

6. Halazy, S.; Ehrhard, A.; Danzin, C. J. Am. Chem. Soc. 1991, 113, 315. doi:10.1021/ja00001a045

7. Beauchamp, L. M.; Tuttle, J. V.; Rodriguez, M. E.; Sznaidman, M. L. J. Med. Chem. 1996, 39, 949. doi:10.1021/jm950736k

8. Kritzyn, A. M.; Komissarov, V. V. Russ. J. Bioorg. Chem. 2005, 31, 609-615.

9. Conant, J. B.; MacDonald, A. D.; Kinney, A. McB. J. Am. Chem. Soc. 1921, 43, 1928. doi:10.1021/ja01441a022

10. Conant, J. B.; Coyne, B. B. J. Am. Chem. Soc. 1922, 44, 2530. doi:10.1021/ja01432a021

11. Gulyukina, N. S.; Dolgina, T. M.; Bondarenko, G. N.; Beletskaya, I. P.; Bondarenko, N. A.; Henry, J.-C.; Lavergne, D.;

Ratovelomanana-Vidal, V.; Genet, J.-P. Russ. J. Org. Chem. 2002, 38, 573. doi:10.1023/A:1016511609369

12. Gulyukina, N. S.; Dolgina, T. M.; Bondarenko, G. N.; Beletskaya, I. P.; Ilyin, M. M.; Davankov, V. A.; Pfaltz, A. Tetrahedron: Asymmetry 2003, 14, 1397. doi:10.1016/S0957-4166(03)00224-6

13. Yu, J.-W.; Huang, S. K. Phosphorus, Sulfur Silicon Relat. Elem. 1997, 131, 107. doi:10.1080/10426509708031600

14. Yu, J.-W.; Shih, C.-C.; Huang, S. K. Phosphorus, Sulfur Silicon Relat. Elem. 1997, 131, 129. doi:10.1080/10426509708031601

15. Laatikainen, R.; Niemitz, M.; Weber, U.; Sundelin, J.; Hassinen, T.; Vepsäläinen, J. J. Magn. Reson. 1996, 120, 1-10. doi:10.1006/jmra.1996.0094 
16. Florio, S.; Troisi, L.; Capriati, V.; Suppa, G. Eur. J. Org. Chem. 2000, 3793.

doi:10.1002/1099-0690(200011)2000:22<3793::AID-EJOC3793>3.0.C O;2-M

17. Yang, X.; Wang, Z.; Fang, X.; Yang, X.; Wu, F.; Shen, Y. Synthesis 2007, 1768. doi:10.1055/s-2007-983705

18. Isaacs, N. S. Physical Organic Chemistry; Longman: Malaysia, 1992; pp 589-647.

\section{License and Terms}

This is an Open Access article under the terms of the Creative Commons Attribution License

(http://creativecommons.org/licenses/by/2.0), which permits unrestricted use, distribution, and reproduction in any medium, provided the original work is properly cited.

The license is subject to the Beilstein Journal of Organic Chemistry terms and conditions:

(http://www.beilstein-journals.org/bjoc)

The definitive version of this article is the electronic one which can be found at: doi:10.3762/bjoc. 6.63 